\title{
Effects of TGF- $\beta 1$ and alginate on the differentiation of rabbit bone marrow-derived mesenchymal stem cells into a chondrocyte cell lineage
}

\author{
WAN-ZONG WANG* ${ }^{*}$ XIAO-DONG YAO*, XIAO-JIN HUANG, JIN-QUAN LI and HAO XU \\ Department of Orthopedics, Fuzhou General Hospital of Nanjing Command, PLA, Fuzhou, Fujian 350025, P.R. China
}

Received October 18, 2014; Accepted May 1, 2015

DOI: $10.3892 /$ etm.2015.2584

\begin{abstract}
The aim of the present study was to investigate the effect of a three-dimensional (3D) culture system of sodium alginate gel on the directional differentiation induction of bone marrow-derived mesenchymal stem cells (BMSCs) into chondrocytes, as well as the in vitro gene transfection technique. The biological characteristics of the passage and proliferation of rabbit BMSCs were investigated under conditions of in vitro monolayer and 3D culture of sodium alginate gel. Transforming growth factor (TGF)- $\beta 1$ gene recombinant adenoviral cosmid vectors and the recombinant adenoviral vector Ad.TGF- $\beta 1$ were constructed, and the effect of Ad.TGF- $\beta 1$ transfection on the differentiation of BMSCs into chondrocytes was investigated. The whole bone marrow rinsing method was used to obtain, separate and purify the rabbit BMSCs, and the in vitro monolayer and $3 \mathrm{D}$ culture of sodium alginate gel were thus successfully and stably established. A safe, stable and efficient method of constructing Ad.TGF- $\beta 1$ TGF- $\beta 1$ gene recombinant adenoviral vectors was established. Following TGF- $\beta 1$ transfection, BMSCs were able to continuously secrete significantly increased amounts of specific extracellular matrix components of chondrocytes, such as collagen II and proteoglycans. Furthermore, the effects in the post-gene transfection 3D culture group were found to be enhanced compared with those in the monolayer culture group. In conclusion, the 3D culture system of sodium alginate gel and in vitro gene transfection exhibited significant induc-
\end{abstract}

Correspondence to: Professor $\mathrm{Hao} \mathrm{Xu}$, Department of Orthopedics, Fuzhou General Hospital of Nanjing Command, PLA, 156 North Xierhuan Road, Fuzhou, Fujian 350025, P.R. China

E-mail: jinquanlicn@163.com

*Contributed equally

Key words: bone marrow-derived mesenchymal stem cells, TGF- $\beta 1$, gene transfection, chondrocytes, three-dimensional culture tive effects on differentiation, which could be used to promote BMSCs to differentiate into chondrocytes.

\section{Introduction}

In recent years, the number of patients with traumatic arthritis, degenerative osteoarthritis and osteoarthritis has significantly increased, and the articular cartilage defects caused by these diseases have become a major cause of disability, seriously affecting the quality of life of the patients. The articular cartilage is an avascular tissue composed of chondrocytes, which mainly depend on the articular synovial fluid and pericellular matrix for their nutrient supply to enable them to perform anaerobic glycolysis and to supply energy. The self-repair ability of cartilage is limited (1); therefore, cartilage tissue engineering has been a focus for significant development (2). There remain, however, numerous problems with cartilage tissue engineering, such as limited resources; insufficient seed cells; limited passage and proliferation capacities; a lack of well-performing stent materials; the failure to effectively, stably and directly induce stem cells to differentiate into cartilage cells; the susceptibility of tissue-engineered cartilage to dedifferentiation; and the difficulties with phenotypic maintenance (3-5).

Bone marrow-derived mesenchymal stem cells (BMSCs) are a class of stem cells derived from the bone marrow tissue that exhibit a strong potential for proliferation and differentiation $(6,7)$. Due to the advantages of BMSCs, such as being widely sourced, easy to obtain, without medical ethics issues and suitable for amplification in in vitro culture, these cells are considered to be the most suitable source of seed cells for bone tissue engineering (8). Currently, there is no uniform method of in vitro culture and directional induction (9). The aims of the present study, therefore, were as follows: i) To attempt to prepare an in vitro three-dimensional (3D) culture to maintain the passage and proliferation capabilities of BMSCs; ii) to use the transforming growth factor (TGF)- $\beta 1$ gene to transfect BMSCs in order to achieve a sustained and stable expression of TGF- $\beta 1$ and enable the observation of the effects of stably expressed TGF- $\beta 1$ on the directional differentiation of BMSCs into chondrocytes (10); and to investigate the conditions and influencing factors that would affect the differentiation of the stem cells into chondrocytes, 
in order to provide the basic theory and methods for the construction of tissue-engineered cartilage.

\section{Materials and methods}

Separation and culture of BMSCs. The whole bone marrow rinsing method was used to obtain bone marrow blood from the long bone cavity of 4 -week-old New Zealand white rabbits obtained from the animal center of Fuzhou General Hospital of Nanjing Command (Fuzhou, China), and a density-gradient centrifugation method was then performed to isolate the BMSCs. The 24 New Zealand rabbits were sacrificed by an intravenous air bolus injection into the ear vein. Following sacrifice, the skin of the four limbs was prepared and locally disinfected with Anerdian (ShanDong LIRCON Medical Technology Incor., Co., Ltd., Dezhou, China), and the skin and tissues of the four limbs were cut to expose the bones. Phosphate-buffered saline (PBS; Sigma-Aldrich, St. Louis, MO, USA) was then used to rinse out the bone marrow using a 20 - $\mathrm{ml}$ syringe at $4^{\circ} \mathrm{C}$, and the cells were centrifuged at $68 \mathrm{x} \mathrm{g}$ and resuspended with $20 \mathrm{ml}$ incomplete Dulbecco's modified Eagle's medium (DMEM; Gibco ${ }^{\mathrm{TM}}$ Life Technologies, Grand Island, NY, USA). The cell suspension was subsequently injected slowly into a centrifuge tube containing $20 \mathrm{ml}$ Ficoll separation solution (Pharmacia Corp., Peapack, NJ, USA) and centrifuged at $27 \times \mathrm{g}$ for $30 \mathrm{~min}$. The cell suspension at the interface was extracted and added into $20 \mathrm{ml}$ incomplete DMEM and centrifuged at $27 \mathrm{x} g$ for $5 \mathrm{~min}$; the BMSC suspension was then prepared following the discarding of the supernatant. The BMSC suspension was seeded in a $75-\mathrm{cm}^{2}$ culture flask $\left(5 \times 10^{4}\right.$ cells $\left./ \mathrm{cm}^{2}\right)$ and cultured at $37^{\circ} \mathrm{C}$ and $5 \%$ $\mathrm{CO}_{2}$ for $24 \mathrm{~h}$. The medium was changed at 24 and $72 \mathrm{~h}$ for the adherent culture to purify the BMSCs; subsequently, the medium was changed once every 3 days until the cells grew to $90 \%$ confluence. Trypsin $(0.05 \%)$ /EDTA $(0.02 \%)$ (Sigma-Aldrich) was used for the digestion and passage. The primary cells were recorded as P0, sequentially followed by $\mathrm{P} 1, \mathrm{P} 2, \mathrm{P} 3$, etc., until the monolayer subculture was completed. The cellular morphological changes and proliferation conditions were observed under an inverted fluorescence phase contrast microscope (Olympus Corp., Tokyo, Japan). This study was approved by the ethics committee of Fuzhou General Hospital of Nanjing Command.

For the 3D culture, P1 BMSCs at $90 \%$ confluence were digested by trypsin and centrifuged. The cell pellets were suspended in low-viscosity sodium alginate (Sigma-Aldrich) solution $(12.5 \mathrm{~g} / \mathrm{l})$ to a concentration of $2 \times 10^{5}$ cells $/ \mathrm{ml}$. The cell suspension was then added dropwise into $102 \mathrm{mmol} / \mathrm{l}$ $\mathrm{CaCl}_{2}$ solution (5 mmol/1 HEPES, pH 7.4; Sigma-Aldrich) using a $5-\mathrm{ml}$ pipette, allowing the condensed beads to gather in the $\mathrm{CaCl}_{2}$ solution for $\sim 10$ min so that each condensed bead would contain $\sim 10,000$ BMSCs. A five-fold $\mathrm{NaCl}$ solution $(0.15 \mathrm{~mol} / \mathrm{l})$ was used to wash the suspension three times; the beads were then inoculated into six $75-\mathrm{cm}^{2}$ culture flasks and cultured at $37^{\circ} \mathrm{C}$ with $5 \% \mathrm{CO}_{2}$. The medium [10\% fetal bovine serum (FBS) containing DMEM (Gibco Life Technologies)] was changed every other day for an 8-week continuous culture. The culture medium was subsequently discarded, and two-fold $50 \mathrm{mmol} / \mathrm{l}$ EDTA (10 mmol/1 HEPES, pH 7.4) was added for the dissolution. Digestion was performed at $37^{\circ} \mathrm{C}$ for $15 \mathrm{~min}$, followed by centrifugation at $239 \mathrm{x}$ g for $10 \mathrm{~min}$; the supernatant was then discarded, and $0.5 \mathrm{ml}$ type II collagenase was added for the minor digestion. The BMSCs were subsequently collected for observation under the inverted fluorescence phase contrast microscope.

Growth curve of the BMSCs. Healthy P1 cells and cells after 1 week of $3 \mathrm{D}$ culture were collected for digestion with $0.05 \%$ trypsin/0.02\% EDTA to make the cell suspension. The cell suspension was then seeded in 10 dishes $(3.5 \mathrm{~cm})$ at a density of $1 \times 10^{4}$ cells/dish. Two dishes were taken for cell counting on days 3, 5, 7, 9 and 11, respectively, and the remaining cells had their culture medium changed once every 3 days. The final cell-counting results were used to establish the cell growth curve.

Construction and identification of vector. Human spleen tissue mRNA provided by Department of General Surgery, Fuzhou General Hospital of Nanjing Command, was extracted and subjected to open reading frame amplification (1,173 bp) according to the GenBank (http://www.ncbi.nlm.nih.gov/genbank/) human TGF- $\beta 1$ gene sequence (gene sequence no. BC000125). The primers used were as follows: TGF- $\beta 1$ forward primer (with the SalI site), CGCGTCGACATGCCGCCCTCCGGGCTG; TGF- $\beta 1$ reverse primer (with the HindIII site), CCAAGCTTCAGC TGCACTTGCAGGAGC. The TGF- $\beta 1$ gene was amplified by the reverse transcription-polymerase chain reaction (RT-PCR) method, and then inserted into the adenoviral genome-containing cosmid vector pAdEasyl (Stratagene; Agilent Technologies, Inc., Santa Clara, CA, USA), and co-transfected into E. coli BJ5183 (Agilent Stratagene) with the recombinant plasmid pShuttleCMV.TGF- $\beta 1$ (Stratagene). The recombinant cosmid pAd.TGF- $\beta 1$ was then generated by homologous recombination. Following the homologous recombination, the liposomal transfection method was applied to transfect the recombinant cosmid adenoviral pAd.TGF- $\beta 1$ into HEK293 cells (American Type Culture Collection, Manassas, VA, USA) to produce the recombinant adenovirus. Five clones of HEK293 cells were selected for second-generation amplification and PCR identification of whether the recombinant adenovirus carried the target gene.

Transfection and identification. Healthy P1 cells and the BMSCs after 1 week of 3D culture were obtained and passaged in a $24-w e l l$ plate. When the coverage reached $70-80 \%$, trypsin was added for digestion. With regard to the $3 \mathrm{D}$ culture, the condensed beads of sodium alginate were digested with EDTA using the aforementioned method, and three wells were randomly selected for cell counting. The recombinant adenovirus green fluorescent protein (Ad.GFP; Gene Therapy Unit, Baxter Healthcare Corporation, Deerfield, IL, USA) adenovirus solution was subsequently added according to the multiplicity of infection (MOI) values of 0, 5, 20, 50, 100 and 200. Each MOI value was set in three parallel wells. The total volume was established as $100 \mu \mathrm{l}$ with PBS; in addition, $1 \mathrm{ml} \mathrm{5 \%}$ FBS-containing DMEM (Gibco Life Technologies) was added to each well. After $1 \mathrm{~h}, 0.4 \mathrm{ml} 5 \%$ FBS-containing DMEM was added for $36-48 \mathrm{~h}$ culture at $37^{\circ} \mathrm{C}$ with $5 \% \mathrm{CO}_{2}$, and the plate was subsequently observed under an inverted fluorescence 
microscope. Three vision fields of each well were observed. The fluorescent cells were counted under a magnification of $\mathrm{x} 400$ to calculate the percentage of fluorescent cells, and the infection efficiency at each MOI was the average percentage value of the fluorescent cells in the three vision fields.

The cells in the experiment were divided into four groups: i) Ad.TGF- $\beta 1$-transfected BMSCs from 3D culture (T3D group); ii) Ad.TGF- $\beta 1$-transfected monolayer-cultured BMSCs (TMC group); iii) Ad.Null empty virus (Stratagene)-transfected BMSCs from 3D culture (ET3D group); and iv) control BMSCs from 3D culture (3D group). The P1 rabbit BMSCs with good growing conditions were used to prepare a cell suspension with a density of $1 \times 10^{6}$ cells $/ \mathrm{ml}$. For the monolayer culture, a $10-\mathrm{cm}$ dish was used, while three $10-\mathrm{cm}$ dishes were used for the $3 \mathrm{D}$ culture. When the cells reached $70-80 \%$ confluence, the recombinant adenovirus Ad.TGF- $\beta 1$ was transfected into the two cell cultures (MOI, 50:1). The ET3D and 3D groups were set as the controls. To each dish, $10 \mathrm{ml} \mathrm{10 \%} \mathrm{FBS-containing}$ DMEM was added, and the dishes were cultured at $37^{\circ} \mathrm{C}$ with $5 \% \mathrm{CO}_{2}$. The medium was replaced once every 3 days.

$R T$-PCR. According to the aforementioned GenBank TGF- $\beta 1$ gene sequence, the RT-PCR method was performed to detect the expression levels of the mRNA, with GAPDH as the reference gene. Two weeks after the transfection, six wells from each experimental group were taken for the extraction of total RNA by the TRIzol ${ }^{\circledR}$ method (Gibco Life Technologies). Following washing with pre-cooled PBS, the NanoDrop ${ }^{\mathrm{TM}} 2000$ ultramicro spectrophotometer (NanoDrop, Wilmington, DE, USA) was used to detect the optical density values at 260 and $280 \mathrm{~nm}$ and calculate the RNA content. Total RNA $(1 \mu \mathrm{g})$ was reverse-transcribed into cDNA according to the instructions of the RT kit (PrimeScript ${ }^{\mathrm{TM}}$ One Step RT-PCR Kit Ver. 2; Takara Biotechnology Co., Ltd., Dalian, China), and this cDNA was then used as the template for the PCR reactions. The PCR reaction conditions were as follows (Applied Biosystems 7500 Real-Time PCR System; Applied Biosystems Life Technologies, Foster City, CA, USA): $95^{\circ} \mathrm{C}$ pre-denaturation for $3 \mathrm{~min} ; 94^{\circ} \mathrm{C}$ denaturation for $40 \mathrm{sec}, 56^{\circ} \mathrm{C}$ annealing for $40 \mathrm{sec}$ and $72^{\circ} \mathrm{C}$ extension for $45 \mathrm{sec}$ (35 cycles); and then final extension at $72^{\circ} \mathrm{C}$ for $10 \mathrm{~min}$. The primer sequences (Sangon Biotech Co. Ltd., Shanghai, China) were as follows: GAPDH upstream primer, 5'-GAAGGTCGGAGTCAACGG-3'; GAPDH downstream primer, 5'-GGAAGATGGTGATGGGATT-3'; TGF- $\beta 1$ upstream primer, 5'-CGCGTCGACATGCCGCCCGG GCTG-3'; TGF- $\beta 1$ downstream primer, 5'-CCAAGCTTC AGCTGCACTTGCAGGAGC-3'. The PCR products were identified by $1.5 \%$ agarose gel electrophoresis; the scanning and analysis of the results were performed by the gel imaging system (Bio-Rad Laboratories, Hercules, CA, USA).

Western blot analysis. According to the aforementioned grouping, the transfected cells were extracted 4 weeks after the transfection; the medium was removed and the cells were washed twice with pre-cooled PBS. The cells were then scraped from the dish with a cell scraper, and the supernatant was discarded following centrifugation. Proteins were extracted from the lysate according to the manufacturer's instructions. Briefly, 100-200 $\mu$ l lysis buffer containing phosphatase inhibitor was added to the collected cells, and incubated in ice for $0.5 \mathrm{~h}$. Subsequently, the supernatant were collected by centrifugation at $20,817 \times \mathrm{g}$ at $4^{\circ} \mathrm{C}$ for $20 \mathrm{~min}$, then stored at $-80^{\circ} \mathrm{C}$. Next, a bicinchoninic acid assay (Bio-Rad Laboratories) was performed to determine the protein content. Following denaturation, the mixture underwent polyacrylamide gel electrophoresis and transfer to polyvinylidene difluoride membranes, which were blocked for $2 \mathrm{~h}$ with $5 \%$ skimmed milk with Tris- $\mathrm{HCl}(20 \mathrm{mmol} / \mathrm{l}), \mathrm{NaCl}(13 \mathrm{mmol} / \mathrm{l})$ and $0.1 \%$ Tween-20 (TBS-T; Sigma-Aldrich). Following washing, the polyclonal TGF- $\beta 1$ primary antibody (cat. no. sc-146; dilution, 1:1,000; Santa Cruz Biotechnology, Inc., Dallas, TX, USA), with polyclonal GAPDH (cat. no. sc-25778; dilution, 1:1,000; Santa Cruz Biotechnology, Inc.) as the control, was added to the membrane for overnight incubation at $4^{\circ} \mathrm{C}$; the membrane was then washed three times with $0.25 \%$ TBS-T, for 5 min each time. The corresponding horseradish peroxidase (HRP)-labeled goat anti-rabbit immunoglobulin G (IgG) secondary antibody (cat. no. sc-2027; dilution, 1:2,500; Santa Cruz Biotechnology, Inc.) was added for incubation at room temperature for $1 \mathrm{~h}$. TBS-T was subsequently used to wash the membrane three times for 10 min each time. An enhanced chemiluminescent solution (1:1) (Advansta, Menlo Park, CA, USA) was added for the scanning and data analysis using the gel imaging system (Bio-Rad Laboratories).

Cartilage differentiation. Cell culture was performed according to the aforementioned grouping. Dexamethasone (Dex; $100 \mathrm{nmol} / \mathrm{l}$ ) and vitamin C (VitC; $50 \mathrm{mg} / \mathrm{l}$; Sigma-Aldrich) were added to the medium for the continuous 4 - to 8 -week induction culture. After the 4-week induction culture, the medium was discarded and the four dishes were placed on ice $\left(0^{\circ} \mathrm{C}\right)$ and rinsed twice with PBS at $0^{\circ} \mathrm{C}$. The cellular changes and the changes in the tissue mass in the $3 \mathrm{D}$ culture dishes were observed under the inverted fluorescence phase contrast microscope.

ELISA for type II collagen and aggrecan. On weeks 1, 2, 4 and 8 post-transfection, the supernatants of the culture media were collected from three wells from each group. The volume of each well was $2 \mathrm{ml}$. When the cells reached $80-90 \%$ confluence, the brain-derived neurotrophic factor standard (Applied Biosystems) and the supernatant were added into a 96-well plate, with three repeated wells for each sample. The assays were carried out according to the manufacturer's instructions (R\&D Systems, Minneapolis, MN, USA).

Immunohistochemical method. Untransfected P1 BMSCs were set as the control group, the cell suspension $\left(1 \times 10^{6} / \mathrm{ml}\right)$ was then inoculated onto the slides of culture dishes for 30-min slide adherence. Next, DMEM was added into the culture dishes for a 3 -h culture at $37^{\circ} \mathrm{C}$ and $5 \% \mathrm{CO}_{2}$. An inverted fluorescence phase contrast microscope (Olympus Corporation) was then used to observe the growth of cells, and the slides were removed when the cells grew almost fused. Similarly, the cells of the TMC group were seeded onto the glass slides, type II collagen immunohistochemical detection was performed on weeks 2, 4 and 6 of induction culture. The technical steps of detection included slice preparation, formalin-fixation and overnight culture with the polyclonal anti-collagen II primary antibody (cat. no. BA1088; dilution, 1:50; Wuhan Boster 

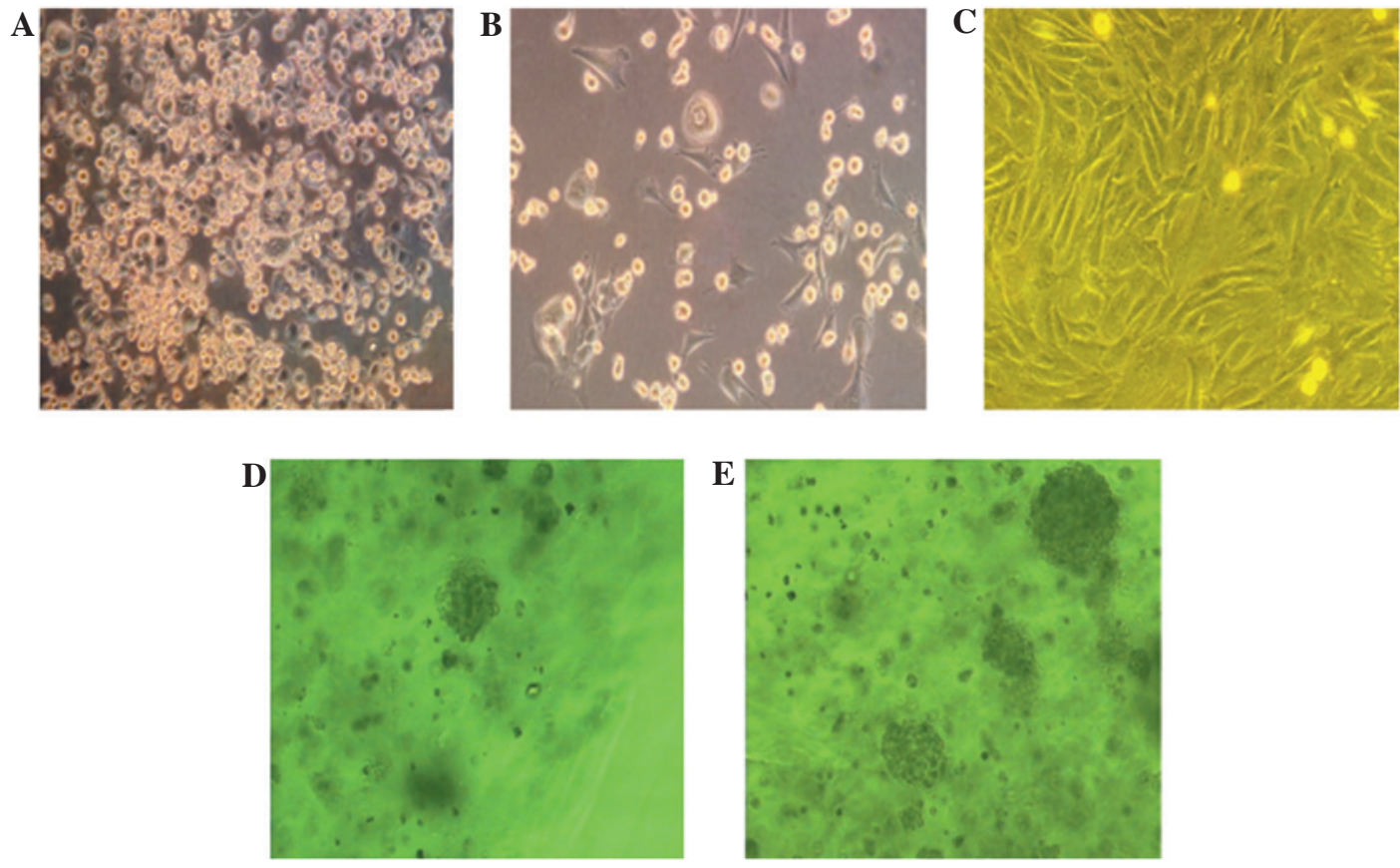

Figure 1. Representative images of primary, subcultured and three-dimensionally-cultured bone marrow-derived mesenchymal stem cells by inverted fluorescence microscopy (magnification, x200). (A and B) Primary culture at (A) 48 h and (B) day 8; (C) subculture at day 10; (D and E) three-dimensional culture at (D) day 7 and (E) 2 months.

Biological Engineering Co., Ltd., Wuhan, China), followed by incubation with the biotinylated goat anti-rabbit $\operatorname{IgG}(1: 200)$ at $37^{\circ} \mathrm{C}$ for $40 \mathrm{~min}$ and streptavidin-HRP (cat. no. BA1003; dilution, 1:200; Wuhan Boster Biological Engineering Co., Ltd.) at $37^{\circ} \mathrm{C}$ for $40 \mathrm{~min}$. Following incubation with streptavidin-HRP, the samples were stained with $0.05 \%$ 3,3'-diaminobenzidine plus $0.03 \% \mathrm{H}_{2} \mathrm{O}_{2}$, counterstained with hematoxylin, washed with $\mathrm{H}_{2} \mathrm{O}$, differentiated with $\mathrm{HCl}-\mathrm{EtOH}$ (2 sec), re-washed with $\mathrm{H}_{2} \mathrm{O}$ and placed in conventional resin.

Statistical analysis. Data are presented as the mean \pm standard deviation for the indicated number of independently performed experiments. Statistical significance was determined by an independent samples $t$-test. $\mathrm{P}<0.05$ was considered to indicate a statistically significant difference. The results were analyzed using SPSS software (version 13.0; SPSS, Inc., Chicago, IL, USA).

\section{Results}

Biological characteristics. After $48 \mathrm{~h}$ of cell culture, the proliferation of the primary adherent cells appeared dispersed and clonal, and the cells exhibited a fibroblast-like spindle shape; 4-5 days later, the adherent cells began to proliferate and transform into triangle, polygonal and spindle shapes. Partial areas subsequently formed unequal-sized colonies; the colonies continued expanding and fusing and the inner cells were morphologically uniform. In the subculture group, the cells were uniformly long-spindled, fusiform and polygonal, and proliferation was rapid; 3 weeks later, the cells began aging and declining. The division and proliferation of the BMSCs in the sodium alginate 3D gel beads were enhanced, with mass- and cluster-like growth and the formation of cell microspheres. The cell microspheres suspended inside the sodium alginate gel remained round in appearance, more passages were performed and the duration was considerably longer; after 6-8 weeks, the cells appeared to age and decline. The cell counts and colonies were significantly higher in the $3 \mathrm{D}$ culture than those in the in vitro monolayer subculture, indicating that the 3D culture system could generate more stable BMSCs (Fig. 1).

The growth curves of the two BMSC cultures were similar; however, the $3 \mathrm{D}$ culture curve exhibited a more rapid and early growth period than the monolayer culture, and the plateau phase was maintained for longer (up to 8 weeks) (Fig. 2).

Transfection and identification. Following transfection, the expression of the green fluorescent protein (GFP) gene in the Ad.GFP-transfected BMSCs resulted in the appearance of green fluorescence under the microscope; by contrast, no fluorescence was observed in the BMSCs that were not transfected with Ad.GFP. The fluoroscopic observation results of the Ad.GFP-transfected BMSCs were as follows: $15.8 \pm 1.1 \%$

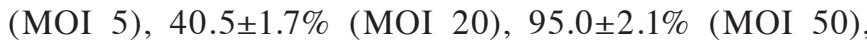
$96.8 \pm 2.4 \%$ (MOI 100) and $98.1 \pm 2.8 \%$ (MOI 200). The results showed that with increasing $\mathrm{MOI}$ values, the ratio of GFP-positive cells also increased. When the MOI was 50, the ratio of green-stained rabbit BMSCs was $>95 \%$, indicating that the in vitro transfection efficiency of the adenovirus was high, and the target gene could be effectively expressed. In order to achieve the optimal dose-effect ratio, an MOI value of 50 was set as the transfection fold for further experiments (Fig. 3).

The RT-PCR and western blot analyses showed that strong TGF- $\beta 1$ expression was present in the cell lysates $48 \mathrm{~h}$ after the transfection; TGF- $\beta 1$ could also be detected at weeks 2 and 4 post-transfection. The two control groups showed no clear bands. The expression levels of TGF- $\beta 1$ mRNA and protein were highest at $\sim 2$ weeks. With the extension 


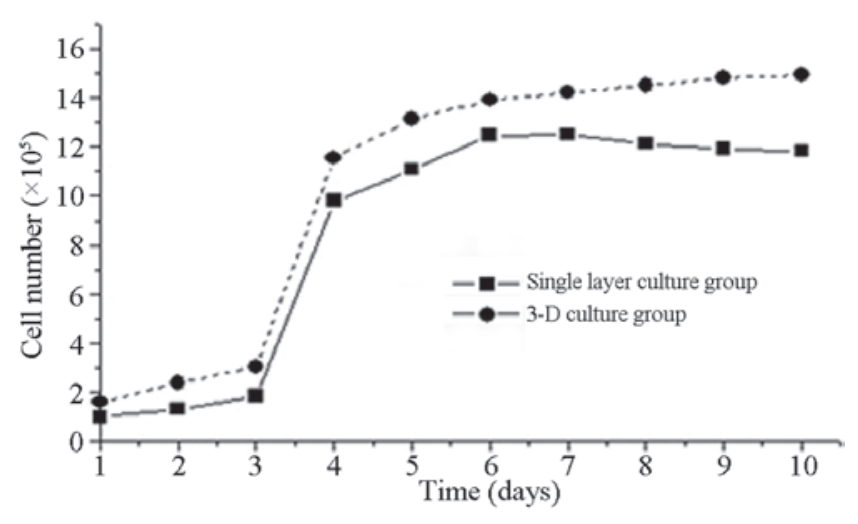

Figure 2. Growth curve of cells in single (mono) layer and 3D culture. 3D, three-dimensional.

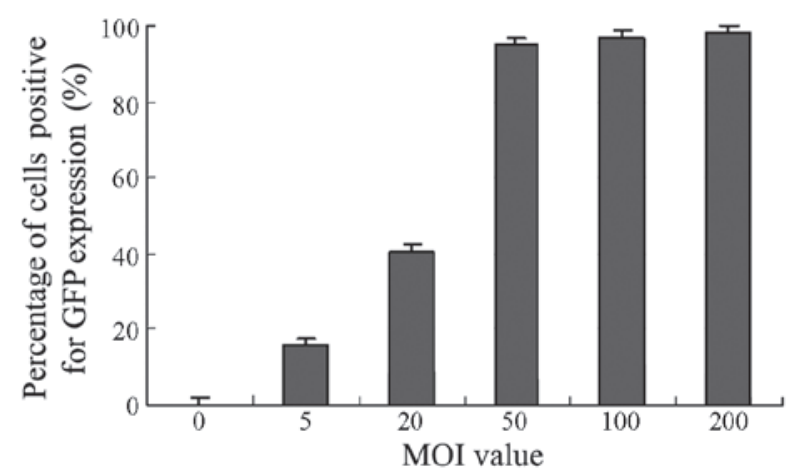

Figure 3. Transfection efficiency of bone-marrow-derived stem cells transfected with adenovirus at different MOI values. GFP, green fluorescent protein; MOI, multiplicity of infection.

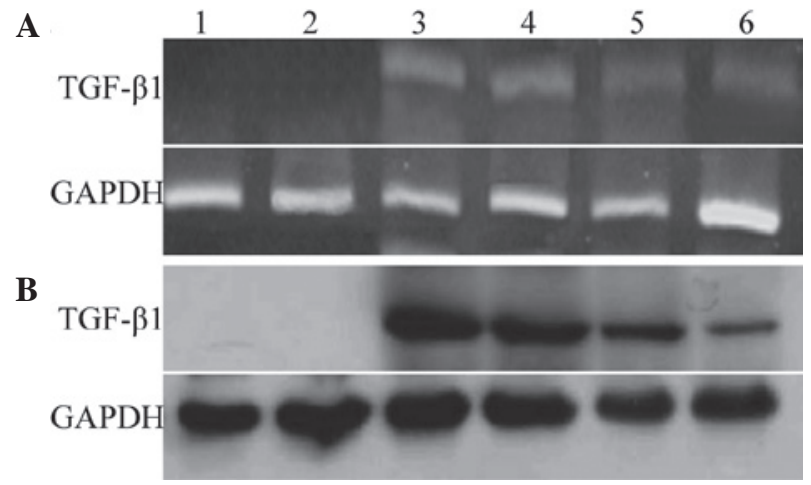

Figure 4. Detection of TGF- $\beta 1$ mRNA and protein expression at different time-points by (A) reverse transcription-polymerase chain reaction and (B) western blotting, respectively. Lane 1, blank control BMSCs from 3D culture; lane 2, ET3D group; lane 3, T3D group (all 2 weeks after transfection); lane 4, T3D group (4 weeks after transfection); lane 5, TMC group ( 2 weeks after transfection); lane 6, TMC group (4 weeks after transfection). GAPDH was used as the internal control. TGF, transforming growth factor T3D, Ad.TGF- 31 -transfected BMSCs from 3D culture; ET3D, Ad.Null empty virus-transfected BMSCs from 3D culture; TMC, Ad.TGF- $\beta 1$-transfected monolayer-cultured BMSCs; BMSC, bone-marrow-derived stem cell; 3D, three-dimensional.

of culture time and generations, the expression of TGF- $\beta 1$ mRNA and protein decreased gradually; among the groups, the relative expression of the T3D group was notably higher than that of the TMC group (Figs. 4A and B), indicating that the TGF- $\beta 1$-carrying recombinant adenovirus could be used to effectively transfect the rabbit BMSCs to elicit high levels of mRNA expression. In addition, the protein expression of the T3D group was evidently stronger than that of the TMC group.

Differentiation promotion. According to the grouping, Dex (100 $\mathrm{nmol} / \mathrm{l})$ and VitC $(50 \mathrm{mg} / \mathrm{l})$ were added for the induction culture. An irregular cell mass could be observed at the bottom of the dish of the T3D group $48 \mathrm{~h}$ later; the diameter of this cell mass became progressively larger, forming small clumps of tissue after 2 weeks. Continued culturing gradually enlarged the tissue mass, which exhibited a pink-white color at 4 weeks (Fig. 5). No tissue clumps formed in the TMC and control groups.

ELISA. At all time-points following transfection, the type II collagen expression in the T3D group was significantly increased compared with that in the TMC group $(\mathrm{P}<0.05)$. The expression in the T3D group reached a peak on day 28 post-transfection $(82.976 \pm 3.452 \mathrm{ng} / \mathrm{ml})$ and then decreased gradually, while that in the TMC group reached its highest point on day 14 post-transfection $(62.331 \pm 3.314 \mathrm{ng} / \mathrm{ml})$ and then steadily declined. The two control groups had almost no expression of type II collagen. On day 56 post-transfection, the type II collagen expression could still be detected in the T3D group, while almost no type II collagen expression was observed in the TMC group; the difference between the groups was statistically significant $(\mathrm{P}<0.05)$ (Fig. 6A). The proteoglycan expression was similar to the type II collagen expression (Fig. 6B).

Immunohistochemistry. Following transfection, the cells in the TMC group at weeks 2 and 4 were seeded onto the slides and subjected to type II collagen immunohistochemical detection. The tissue clumps of the T3D group at weeks 2, 4 and 6 were prepared as frozen sections and then utilized for type II collagen immunohistochemical detection. The results for the expression of type II collagen were positive in the TMC group at weeks 2 and 4 (stained brown), while immunohistochemistry at week 6 did not detect type II collagen expression. In the T3D group, the type II collagen immunohistochemistry at weeks 2, 4 and 6 revealed strong, positive expression (stained yellow-brown); the continued positive result at the 6 th week indicated that the expression of type II collagen could still be detected. No type II collagen expression was found in the untransfected BMSC control group (Fig. 7).

\section{Discussion}

The principles of cell biology indicate that the number and density of cells play an important role in the proliferation and differentiation of BMSCs. During the development of cartilage, the aggregation of numerous local mesenchymal cells can be observed, forming a higher cell density; this cell aggregation has significance toward the differentiation of BMSCs into cartilage (11). Through the in vitro culture of BMSCs, Nakahara et al (12) found that, when the cells reached a certain density, BMSCs could differentiate into chondrocytes. A study confirmed that the BMSCs passaged with a higher density could 

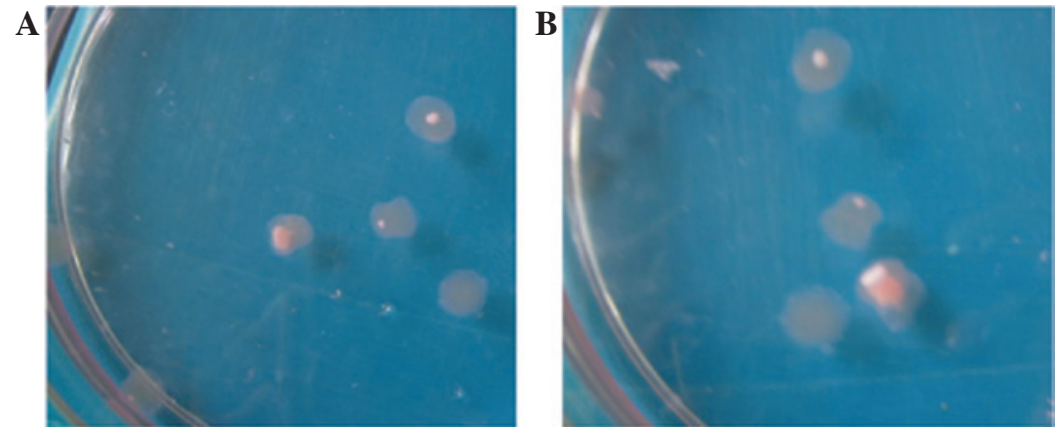

Figure 5. Cartilage tissue clumps of the T3D group at weeks (A) 2 and (B) 4. T3D, Ad.TGF- $\beta 1$-transfected 3D-cultured bone marrow-derived stem cells.
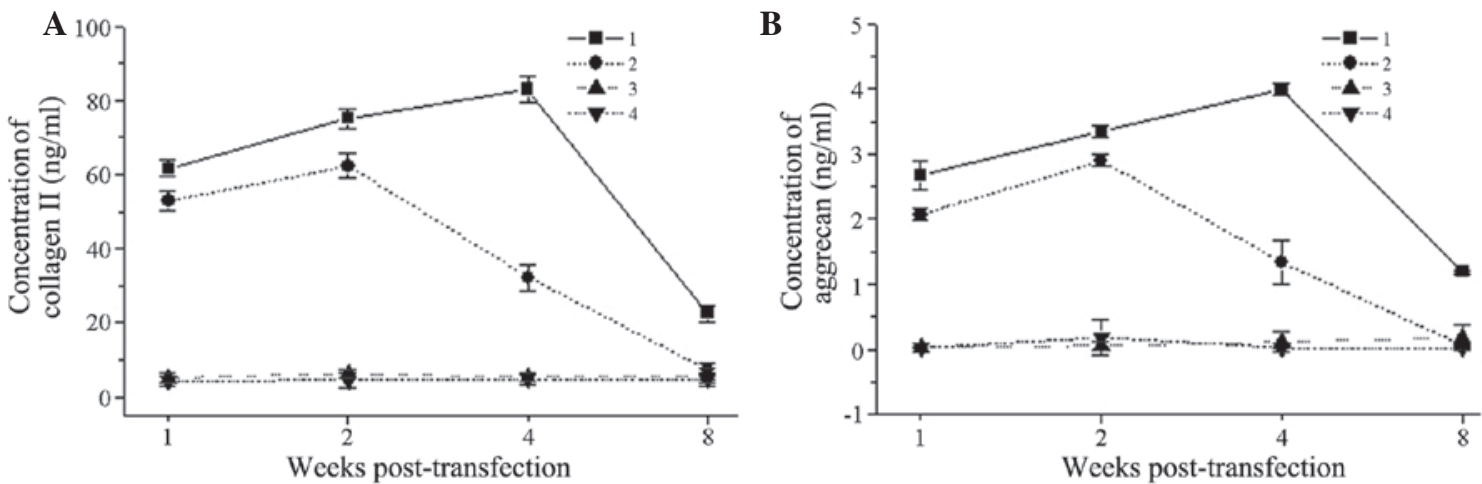

Figure 6. Content detection of (A) type II collagen and (B) proteoglycan (aggrecan) in the supernatant of TGF- $\beta 1$-transfected rabbit BMSCs at different time-points by ELISA. The two control groups exhibited similar results and had almost no expression of type II collagen. At all time-points, the type II collagen expression in the T3D group was significantly higher than that in the TMC group $(\mathrm{P}<0.05)$. The proteoglycan expression was similar to the type II collagen expression. Results are presented as mean \pm standard deviation, $\mathrm{n}=3$. TGF, transforming growth factor; BMSC, bone-marrow-derived stem cell; group 1, T3D group (Ad.TGF- $\beta 1$-transfected BMSCs from 3D culture); group 2, TMC group (Ad.TGF- $\beta 1$-transfected monolayer-cultured BMSCs); group 3, ET3D group (Ad.Null empty virus-transfected BMSCs from 3D culture); group 4, 3D group (control BMSCs from 3D culture); 3D, three-dimensional.
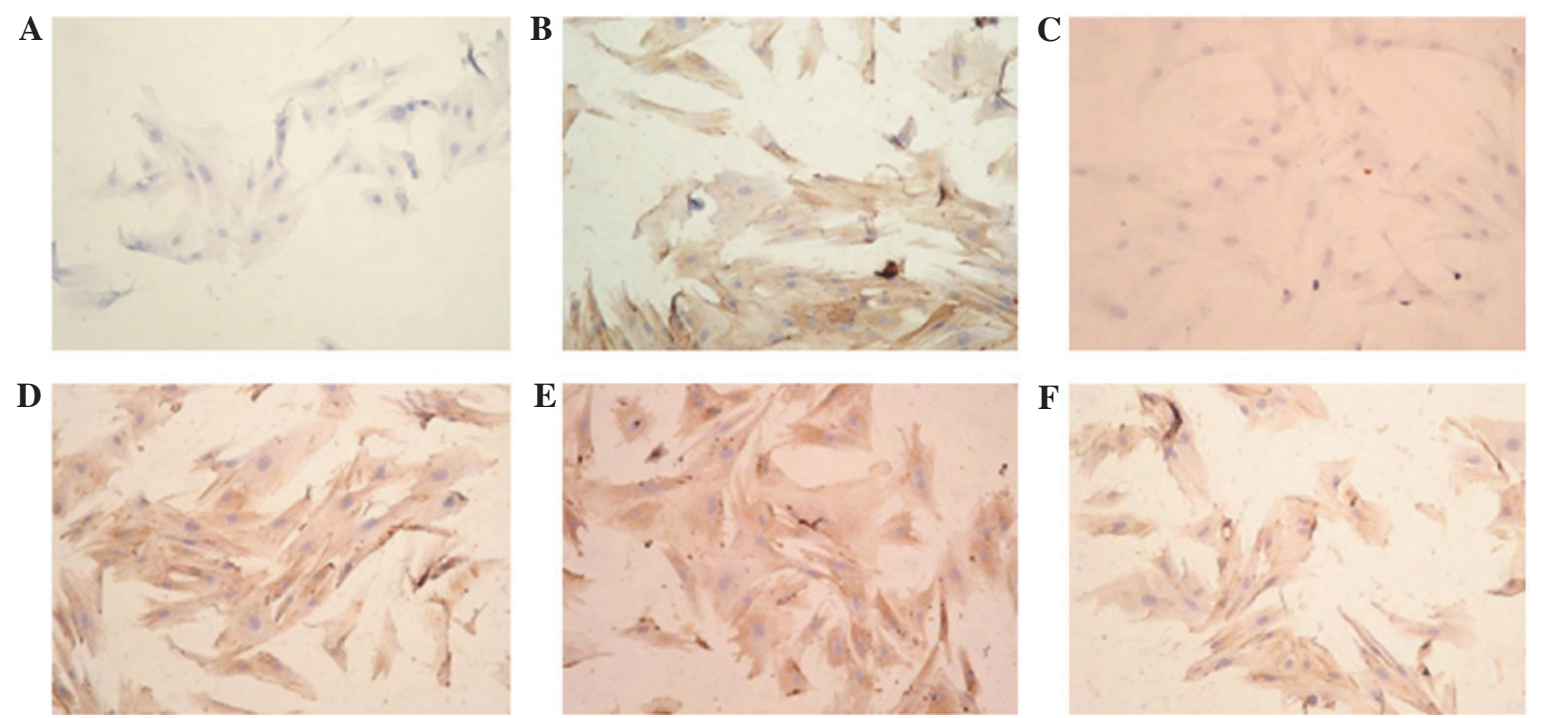

Figure 7. Type II collagen immunohistochemical results for BMSCs in the TMC and T3D groups following transfection (the untransfected group was set as the control). (A) Control group (-); (B) TMC group after 2 weeks (+); (C) TMC group after 6 weeks (-); (D) T3D group after 2 weeks (+); (E) T3D group after 4 weeks (+); (F) T3D group after 6 weeks (+). BMSC, bone-marrow-derived stem cell; T3D, Ad.TGF- $\beta 1$-transfected BMSCs from 3D culture; ET3D, Ad.Null empty virus-transfected BMSCs from 3D culture; TMC, Ad.TGF- $\beta 1$-transfected monolayer-cultured BMSCs; TGF, transforming growth factor; 3D, three-dimensional.

be differentiated into chondrocytes, expressing the chondrocyte phenotype, and would, therefore, form cartilage nodules when implanted into nude mice. By contrast those passaged with low density would fail to differentiate into chondrocytes (13), indicating that certain cell densities were necessary for the differentiation of BMSCs into chondrocytes. However, compared with 
the other types of cells inside the bone marrow, the abundance of BMSCs inside the bone marrow is not high, with the ratio being 0.01-1:1 $\times 10^{4}$ among the bone marrow cells (14).

The construction of a good in vitro culture system would not only facilitate the proliferation of BMSCs, so that the seed cells could be obtained at the required scale, but would also be of use for the differentiation of BMSCs into cartilage. Currently, the in vitro BMSC culture system has no consistent standard: The common culture methods of BMSCs are two-dimensional (2D) culture (including very low-density subculture, low oxygen tension and gene transfection cultures), 3D culture, and inactive and active cell cultures (15). At present, the method of $2 \mathrm{D}$ culture is most widely adopted. Although 2D culture techniques are widely used and gradually improving, they exhibit the following shortcomings, which are challenging to overcome: i) The surface area of the adherent cells is limited; therefore the cell yield is low; ii) the aseptic process is cumbersome, and would be contaminated easily; iii) the metabolites gradually accumulate, and untimely exclusion would lead to the poor growth of cells and even degeneration; iv) the cells remaining in the in vivo $3 \mathrm{D}$ structure with the extracellular matrix would show alterations in biological behavior, and variation would easily occur. Therefore, the experimental study of the application of $3 \mathrm{D}$ cell-culture techniques into cartilage tissue engineering has been initiated.

In 1998, Qiu et al (16) performed a 3D BMSC culture. Glowacki et al (17) used a collagen sponge as the carrier for the $3 \mathrm{D}$ perfusion culture, which resulted in a reduction in the accumulation of metabolites; the results revealed that the content of extracellular matrix was increased and the activities and functions of the cultured cells were strengthened. Angele et al (18) applied in vitro-amplified human BMSCs into a hyaluronic acid-gelatin complex sponge, and a TGF- $\beta 1$-exterior chemical limited medium (Dex, VitC, etc.) was added. After 28 days, cartilage tissue was produced. Ponticiello et al (19) applied human BMSCs to a gelatin sponge, with the addition of TGF- $\beta 3$, and succeeded in culturing chondrocytes. Compared with the $2 \mathrm{D}$ culture, the $3 \mathrm{D}$ culture was able to accommodate high-density cell adhesion and proliferation, was favorable for intracellular signaling conduction and could provide a suitable microenvironment to maintain the metabolic activities of cells. In addition, a 3D culture facilitates the increased synthesis of extracellular matrix components, enhances the activities and functions of the cultured cells and enables the extracellular matrix to be fixed near the cells, which prevents it from being lost into the medium as easily as that in the monolayer culture; the $3 \mathrm{D}$ culture system therefore has important regulatory roles in cell proliferation, differentiation and metabolism. Currently, the biological material used in 3D culture can take numerous forms, the most common being the gelatin sponge, hyaluronic acid-gelatin complex sponge, collagen sponge and alginate beads (20). Since alginate is a linear polysaccharide with good biocompatibility and can be metabolized in vivo through hydrolytic and enzymatic pathways, its metabolites do not generate adverse effects toward the cells (21). Furthermore, it can undergo ionic gelation in the presence of $\mathrm{Ca}^{2+}$, exhibiting mechanical properties similar to those of normal cartilage tissue, thereby better maintaining the phenotype of the seed cells. The plasticity of alginate is also strong; thus, it may be an optimum 3D culture material (22). In the present study, a
3D culture system of sodium alginate gel was constructed, and the in vitro amplification of BMSC seed cells was successfully performed. The experimental results showed that, compared with the 2D culture, the BMSCs obtained from the 3D culture exhibited a more rapid and early growth, a more evident increase in total number, a longer maintenance time and a longer plateau phase (up to 8 weeks).

The sodium alginate $3 \mathrm{D}$ culture system constructed in the present experiment was not only favorable for BMSC proliferation, generating seed cells at the required scale, but also aided the differentiation of the BMSCs into cartilage. In the $3 \mathrm{D}$ culture conditions, the adenoviral vector was capable of transfecting BMSCs successfully in vitro (23), and cartilage tissue clumps could be obtained successfully 4 weeks after the induction of the culture. Western blot analysis was performed to detect the expression levels of TGF- $\beta 1$ protein following the transfection, and RT-PCR analysis was performed to detect the transcription levels of TGF- $\beta 1$ mRNA. The results showed that the expression levels of TGF- $\beta 1$ protein and mRNA in the $3 \mathrm{D}$ group were significantly higher than those in the TMC and control groups, indicating that the transfection could achieve the sustained and effective expression of TGF- $\beta 1$ and induce the formation of cartilage tissue in conjunction with other stimuli (e.g. Dex and VitC) (24). The detection of cartilage extracellular matrix secretion products indicated that the TGF- $\beta$-transfected BMSCs were capable of secreting such cartilage-specific matrix components as type II collagen and proteoglycans. The secretion level in the T3D group was higher than that in the TMC group, and the duration of the type II collagen secretion was longer, indicating that the transfected BMSCs exhibited in vitro differentiation into chondrocytes, with the T3D group performing in a superior way to the TMC group.

An explanation for the aforementioned results may be that the sodium alginate $3 \mathrm{D}$ culture system was able to accommodate high-density cell adhesion and proliferation, which was favorable for intracellular signal transduction. The use of sodium alginate may also have reduced the accumulation of metabolic products, providing a suitable microenvironment that was able to maintain the metabolic activities of the cells and to form the 3D structure together with the extracellular matrix. The condensed alginate beads provided a 3D microenvironment that was similar to the in vivo conditions, reducing the variation in the biological behavior of the cells. Furthermore, the content of the extracellular matrix was improved and could be fixed near the cells, instead of becoming immersed in the culture solution; thus, the cells were surrounded by rich extracellular matrix. The 3D culture system therefore played an important regulatory role in the cell proliferation, differentiation and metabolism.

The present experimental study completed the separation and purification of rabbit BMSCs and successfully established monolayer and 3D cultures in vitro. Compared with the monolayer culture, the proliferation of the BMSCs in the 3D culture was more active, the number of passages was increased and the duration of the culture was longer. After 6-8 weeks, the cells appeared to be aging and declining. Importantly, the 3D culture system was more comparable with the microenvironment in vivo, which has laid a foundation for the study of BMSCs in animal models. The recombinant adenovirus Ad.TGF- $\beta 1$ was 
transfected into the BMSCs, and it was found that the BMSCs in the monolayer and 3D cultures had a high level of TGF- $\beta 1$ expression following transfection, as detected by RT-PCR and western blotting. TGF- $\beta 1$ expression continued until 6 weeks after the transfection. The result indicated that the transfection of BMSCs by the recombinant adenovirus Ad.TGF- $\beta 1$ was safe and efficient. The protein expression in the cells in $3 \mathrm{D}$ culture was evidently superior to that in the cells in monolayer culture. Following the RT-PCR and western blot analyses, ELISA was used to detect the presence of type II collagen and proteoglycan in the supernatant of the culture after TGF- $\beta 1$ transfection, as a measure of the BMSC differentiation into cartilage cells. The results revealed that, following TGF- $\beta 1$ transfection, the BMSCs could continuously secrete such specific extracellular matrix components of chondrocytes as type II collagen and proteoglycans; the levels of the these components were found to have increased significantly, and the post-gene transfection $3 \mathrm{D}$ culture group exhibited superior results to the monolayer culture group. In conclusion, the present series of experimental results has shown that the use of a 3D culture system and gene transfection in vitro can promote BMSC proliferation and aid the differentiation of BMSCs into chondrocytes.

\section{References}

1. O'Driscoll SW: The healing and regeneration of articular cartilage. J Bone Joint Surg Am 80: 1795-1812, 1998.

2. Awad HA, Butler DL, Boivin GP, Smith FN, Malaviya P, Huibregtse B and Caplan AI: Autologous mesenchymal stem cell-mediated repair of tendon. Tissue Eng 5: 267-277, 1999.

3. Ostrander RV, Goomer RS, Tontz WL, Khatod M, Harwood FL, Maris TM and Amiel D: Donor cell fate in tissue engineering for articular cartilage repair. Clin Orthop 389: 228-237, 2001.

4. Colther DC, Sekiya I and Prockop DJ: Identification of a subpopulation of rapidly self-renewing and multipotential adult stem cells in colonies of human marrow stromal cells. Proc Natl Acad Sci USA 98: 7841-7845, 2001.

5. Huang JI, Zuk PA, Jones NF, Zhu M, Lorenz HP, Hedrick MH and Benhaim P: Chondrogenic potential of multipotential cells from human adipose tissue. Plast Reconstr Surg 113: 585-594, 2004.

6. Meirelles Lda S,Fontes AM, Covas DT and Caplan AI: Mechanisms involved in the therapeutic properties of mesenchymal stem cells. Cytokine Growth Factor Rev 20: 419-427, 2009.

7. BooL, Selvaratnam L, Tai CC, Ahmad TS and KamarulT: Expansion and preservation of multipotentiality of rabbit bone-marrow derived mesenchymal stem cells in dextran-based microcarrier spin culture. J Mater Sci Mater Med 22: 1343-1356, 2011.

8. Seong JM, Kim BC, Park JH, et al: Stem cells in bone tissue engineering. Biomed Mater 5: 062001, 2010.
9. Augello A, Kurth TB and De Bari C: Mesenchymal stem cells: A perspective from in vitro cultures to in vivo migration and niches. Eur Cell Mater 20: 121-133, 2010.

10. Worster AA, Nixon AJ, Brower-Toland BD and Williams J: Effect of transforming growth factor betal on chondrogenic differentiation of cultured equine mesenchymal stem cells Am J Vet Res 61: 1003-1010, 2000.

11. Yoo JU, Barthel TS, Nishimura K, et al: The chondrogenic potential of human bone-marrow-derived mesenchymal progenitor cells. J Bone Joint Surg Am 80: 1745-1757, 1998.

12. Nakahara H, Dennis JE, Bruder SP, Haynesworth SE, Lennon DP and Caplan AI: In vitro differentiation of bone and hypertrophic cartilage from periosteal-derived cells. Exp Cell Res 195: 492-503, 1991.

13. Bruder SP, Fink DJ and Caplan AI: Mesenchymal stem cells in bone development, bone repair and skeletal regeneration therapy. J Cell Biochem 56: 283-294, 1994.

14. Mostafa NZ, Uludağ H, Varkey M, Dederich DN, Doschak MR and El-Bialy TH: In vitro osteogenic induction of human gingival fibroblasts for bone regeneration. Open Dent J 5: 139-145, 2011.

15. Lisignoli G, Remiddi G, Cattinil L, et al: An elevated number of differentiated osteoblast clonies can be obtained from rat bone marrow stromal cells using a gradient isolation procedure. Connect Tissue Res 42: 49-58, 2001.

16. Qiu Q, Ducheyne P, Gao H and Ayyaswamy P: Formation and differentiation of three-dimensional rat marrow stromal cell culture on microcarriers in a rotating-wall vessel. Tissue Eng 4: 19-34, 1998.

17. Glowacki J, Mizuno S and Greenberger JS: Perfusion enhances functions of bone marrow stromal cells in three-dimensional culture. Cell Transplant 7: 319-326, 1998.

18. Angele P, Kujat R, Nerlich M, Yoo J, Goldberg V and Johnstone B: Engineering of osteochondral tissue with bone marrow mesenchymal progenitor cells in a derivatized hyaluronan-gelatin composite sponge. Tissue Eng 5: 545-554, 1999.

19. Ponticiello MS, Schinagl RM, Kadiyala S and Barry FP: Gelatin-based resorbable sponge as a carrier matrix for human mesenchymal stem cells in cartilage regeneration therapy. J Biomed Mater Res 52: 246-255, 2000.

20. Majumdar MK, Banks V, Peluso DP and Morris EA: Isolation, characterization and chondrogenic potential of human bone marrow-derived multipotential stromal cells. J Cell Physiol 185: 98-106, 2000.

21. Diduch DR, Jordan LC, Mierisch CM and Balian G: Marrow stromal cells embedded in alginate for repair of osteochondral defects. Arthroscopy 16: 571-577, 2000.

22. Ma HL, Hung SC, Lin SY, Chen YL and Lo WH: Chondrogenesis of human mesenchymal stem cells encapsulated in alginate beads. J Biomed Mater Res A 64: 273-281, 2003.

23. Hwang NS, Im SG, Wu PB, et al: Chondrogenic priming adipose-mesenchymal stem cells for cartilage tissue regeneration. Pharm Res 28: 1395-1405, 2011.

24. Taipaleenmäki H, Harkness L, Chen L, et al: The crosstalk between transforming growth factor- $\beta 1$ and delta like- 1 mediates early chondrogenesis during embryonic endochondral ossification. Stem Cells 30: 304-313, 2012. 